\title{
LAS ARTISTAS CALLEJERAS EN LA FEMINIZACIÓN GRÁFICA DEL ESPACIO DE CIUDAD JUÁREZ
}

\section{Sergio Raúl Recio Saucedo}

Universidad Autónoma de Ciudad Juárez (México)

\section{Resumen}

En el artículo se explican las acciones que realizan las artistas callejeras para contribuir en la feminización de los espacios públicos de Ciudad Juárez puesto que han experimentado relaciones sociales de carácter misógino que afectan las maneras de desenvolverse en el territorio fronterizo. Por ello, las artistas callejeras han desarrollado actividades asociadas con la colocación de paste $u p^{1}$ y la creación de murales para subvertir las dinámicas del patriarcado y empezar a reducir simbólicamente la violencia de género en el ámbito privado y público. Esto, fue analizado bajo los estudios urbanos de género para situar las vivencias de las mujeres dentro de Juárez, ya que sus acciones responden a los procesos sociales de la frontera.

\section{Palabras claves: ARTISTAS CALLEJERAS; MUJERES; CIUDAD JUÁREZ (MÉXICO); FEMINIZACIÓN; ESPACIO PÚBLICO; PASTE UP}

\section{STREET ARTISTS IN THE GRAPHIC FEMINIZATION OF THE CIUDAD JUÁREZ SPACE}

\section{Abstract}

The article explains the actions carried out by street artists to contribute to the feminization of the public spaces of Ciudad Juárez since they have experienced misogynistic social relationships that affect the ways of developing in the border territory. Therefore, street artists have developed activities associated with the placement of paste up and the creation of murals to subvert the dynamics of patriarchy and symbolically begin to reduce gender violence in the private and public spheres. This was analyzed under urban gender studies to situate women's experiences within Juárez, since their actions respond to the social processes of the border.

Keywords: STREET ARTISTS; WOMEN; CIUDAD JUÁREZ (MEXICO): FEMINIZATION; PUBLIC SPACE; PASTE UP

\footnotetext{
Recio Saucedo, Sergio Raúl. 2020. "Las artistas callejeras en la feminización gráfica del espacio de Ciudad Juárez". AusArt 8 (1): 145167. DOI: 10.1387/ausart.21600
}

\section{AUSART}


En el presente artículo se analizan las actividades de intervención que elaboran las grafiteras, las artistas y las muralistas para feminizar de manera gráfica los espacios públicos de Ciudad Juárez. Esta idea, surge por creer que el entorno urbano de Juárez posee una carga negativa a causa de la problemática de los feminicidios convirtiéndolo en inseguro para las mujeres. Además, se considera que la construcción del espacio se ha centrado en satisfacer los requerimientos de la industria maquiladora de exportación, dejando de lado diferentes subjetividades como la femenina. Es decir, se ha construido la ciudad con base a las "necesidades de un ciudadano abstracto (generalmente pensadas en función de hombres, jóvenes y sin problemas de movilidad) no reparan en otras maneras distintas de 'ser' en el espacio: ser mujer, ser anciana, ser niño, ser migrante, ser gay, ser trans” (De Simone 2018, 230).

Bajo este contexto, el artículo se estructura en cinco subtemas que se asocian con: 1. La mujer en el espacio público, donde se analiza el papel que posee la figura femenina en los procesos de urbanización de las ciudades. 2. Feminización de los espacios, donde se explica el término feminización y es vinculado a los espacios. En los apartados 3, 4 y 5 se analizan las actividades que desarrollan las grafiteras, las artistas y las muralistas para colaborar en la feminización gráfica de los espacios de Ciudad Juárez.

\section{EL GÉNERO Y LOS ESPACIOS PÚBLICOS}

La existencia de espacios públicos dentro de las ciudades ha permitido teóricamente el desarrollo de encuentros y de actividades que expresan signos de libertad puesto que los ciudadanos se apropian del carácter público para hacer valer las garantías individuales y colectivas de los espacios. Aquí, las personas emplean los espacios según sus necesidades y posibilidades, ya que observan diversos usos sociales en el territorio. De ello, se desprende una pregunta que se relaciona con saber ¿la configuración actual de los espacios públicos condiciona el libre acceso a las diferentes identidades citadinas? Una respuesta, se encuentra en los estudios urbanos de género porque ayudan al entendimiento sobre las nociones que configuran los espacios de las urbes.

En este sentido, los estudios urbanos de género aparecieron como un campo teórico y una "herramienta conceptual para explicar cómo se han construido las diferencias entre lo femenino y lo masculino en las formas de hacer ciudad" 
(Soto 2018, 17). Es decir, comenzaron a prestar atención a las configuraciones urbanas que limitan la estancia de las mujeres en los espacios públicos al no adecuarse a las necesidades fisiológicas del colectivo femenino. Más bien, se enfocan en las categorías culturales que definen las identidades de las mujeres y los hombres en las urbes.

Las identidades de los géneros son el resultado del "conjunto de prácticas, creencias, representaciones y prescripciones sociales que surgen entre los integrantes de un grupo humano en función de una simbolización de la diferencia anatómica entre hombres y mujeres" (Lamas 2000, 3). Esto, ha generado que el espacio no se adecue a las necesidades del colectivo femenino puesto que es vinculado a una serie de ideas espaciales que las relegan con lo privado, la reproducción y la inmovilidad; mientras, que lo masculino es asociado con lo público, la producción y la movilidad.

Por lo tanto, los espacios públicos han sido configurados por una visión androcéntrica y capitalista que ha permeado los procesos de urbanización de las ciudades al otorgar prioridad a las dinámicas laborales y masculinas ocasionado la perpetuación de los roles de género en la sociedad. Esto, supone "una valoración diferente de las personas y de las actividades que llevan a cabo, al atribuir un valor superior a lo masculino y público y devaluar las tareas relacionadas con el cuidado de las personas y del hogar" (Ortiz 2017, 6).

La diferenciación de lo masculino y femenino genera un proceso de homogenización de los espacios puesto que no permite la otredad y unifica la base material y humana dentro de la productividad. Esta idea concibe al equipamiento urbano como una estructura para el desarrollo de las dinámicas laborales y a los habitantes los considera como usuario sin identidad al presentarse una mecánica funcionalista que uniforma las actividades que se realizan en la ciudad.

El funcionalismo ocasiona un proceso de especialización urbana debido a que "a cada espacio se le atribuyen unas funciones y actividades concretas y donde las actividades productivas son priorizadas en el diseño urbano, invisibilizando las necesidades de la esfera reproductiva" (Valdivia 2018, 66). Esto, hace invisibles a ciertas personas, grupos y etnias, y, potencializa las relaciones de desigualdad al contener sistemas urbanos que no se adaptan a las demandas de los habitantes. Por lo tanto, el acceso público a la sociedad termina siendo restringido al generarse dinámicas de exclusión que perjudica el libre tránsito, la libertad de asociación, de manifestación y de participación ciudadana. 
Ante la exclusión espacial los estudios de género proponen alejarse de la postura dicotómica del género y centrarse en la experimentación de los espacios según su edad, etnia, religión, discapacidad, preferencias. Por lo tanto, la perspectiva de género "aporta una visión amplia de las personas al plantear que las mujeres y los hombres viven y experimentan el espacio de maneras diferentes. Hoy, ambos asumen distintas responsabilidades y esto se materializa diariamente en su relación con el entorno" (Ortiz 2017, 6). Es decir, no existe una sola forma de experimentar los espacios públicos de las urbes, ya que cada una de las identidades tiene distintas necesidades de actuación y de apropiación que deberían ser consideradas para una configuración urbana heterogénea.

Además, Los estudios de género se presentan como un cuerpo teórico que cuestiona el modelo tradicional del espacio público mediante propuestas que colocan en el centro del debate los procesos de segregación y de homogeneización que marginan a la mujer del ámbito público. Con ello, se busca situar a la figura femenina dentro de las dinámicas urbanas para la restructuración de los paradigmas que predominan la configuración de las ciudades y las condicionan al espacio privado. Además, para conseguir un "ejercicio pleno, libre y autónomo de la ciudadanía femenina materializada en: diversas formas de usufructo equitativo, [...] de los bienes y las oportunidades que la ciudad ofrece; la incidencia femenina en la construcción colectiva y participativa de los asuntos de ciudad" (Milena 2012, 107).

El abordaje del espacio público bajo la perspectiva de género y feminista permite pensar en su reconstrucción desde una óptica que se aleja del androcentrismo, la neutralidad y la especialización para centrarse en ideas asociadas con la diversidad, la apertura y la inclusión. La democratización de las ciudades posibilitaría la participación activa de las diferentes identidades ciudadanas eliminando las relaciones sociales selectivas que ha dominado las actividades públicas que perjudican los procesos de interacción y de apropiación de los espacios al limitar y condicionar la utilización de la infraestructura. Con esto, se busca la feminización de los espacios, es decir, la creación de un urbanismo inclusivo que facilite la movilidad y la seguridad femenina mediante la integración de las tareas reproductivas en la configuración urbana. Para ello, "los espacios públicos y los servicios deben responder a las necesidades de todas las personas y facilitar todas las tareas" (Nadurille 2007, 39). 


\section{FEMINIZACIÓN DE LOS ESPACIOS}

La comprensión que se hace de los espacios públicos desde los estudios urbanos de género permite considerar nuevos paradigmas que se alejan del androcentrismo y del capitalismo para centrarse en ideas asociadas con la diversidad, la movilidad y la convivencia como modelos de configuración urbana. Lo anterior, implica el planteamiento de una pregunta que se relaciona con saber ¿cómo son los procesos de urbanización que configuran espacios públicos incorporando las necesidades sociales de las minorías? Una respuesta se encuentra en el entendimiento del término feminización, ya que ayuda a visibilizar los elementos conceptuales que integran la perspectiva de la mujer en las dinámicas urbanas.

En este sentido, el término feminización se vincula con el "proceso de la adquisición de atributos y características propias o asociadas a la mujer; o bien a la adhesión significativa de un número de personas de este género a una situación determinada que en un momento anterior había tenido una composición fundamentalmente masculina" (Otero 2012, 1400). La definición propone una dinámica de cambio físico, simbólico o visual de un objeto, una estructura, un suceso o una formación social que se presenta al interior de las sociedades. Ello, supone para la feminización de los espacios una modificación en la base material de las urbes para transformarlas en construcciones arquitectónicas que posean características utilitarias destinadas a la diversidad cultural.

El proceso de feminización de los espacios públicos, en primer lugar, implicaría comenzar por un cambio estructural que supere la invisibilidad de la mujer dentro de la esfera pública mediante la concatenación de las actividades productividad con las tareas reproductividad. La vinculación sería la respuesta que integra en la ciudad las necesidades de la diversidad social al mejorar las dinámicas de movilidad, de cuidado y de accesibilidad, lo cual promovería la incorporación del colectivo femenino a los espacios. Ello, supone la configuración de una realidad urbana más fluida que se distinga por la conectividad entre los ámbitos personales, públicos y domésticos para la construcción de infraestructura que beneficie a la esfera privada como en el transporte público.

En segundo lugar, supone una modificación en los usos sociales de la configuración urbana al complementar los procesos laborales y mercantiles con las dinámicas domésticas y de cuidado para conseguir un espacio público que asegure la sostenibilidad de la diversidad de la vida en sociedad. El cambio de paradigma es fundamental para "revertir la posición que tienen socialmente y 
para ello es imprescindible reconfigurar los espacios y los tiempos de la ciudad, pensándolos para poder desarrollar todas las actividades de cuidados" (Valdivia 2018, 77). Además, es trascendental para el desarrollo de dinámicas más equitativas donde prime el factor humano sobre el productivo para que las personas puedan elegir, interactuar o moverse dentro de urbes.

En tercer lugar, implica colocar a las personas en el centro del debate de la configuración urbana para considerar la diversidad cultural y romper con "la estandarización de sujetos, cuerpos, vivencias y deseos. Los espacios deben ser flexibles y adaptarse a las diferentes necesidades de las personas y no que las personas se adapten a las condiciones del espacio" (Valdivia 2018, 79). Desde esta perspectiva los procesos de urbanización estarían pensados en las experiencias de los habitantes para satisfacer las necesidades de iluminación, movilidad y seguridad, lo cual generaría una participación activa de la mujer en la apropiación y la intervención de los espacios públicos, lo que dotaría de nuevas vivencias a la urbe.

Por lo tanto, la feminización de los espacios públicos fomenta una dinámica de accesibilidad social al considerar la diversidad como principal elemento para la configuración de los espacios, ya que visibiliza distintas identidades que poseen diferentes capacidades, destrezas y necesidades. La perspectiva femenina proporciona a las personas "espacios equipados para el ocio y la diversidad de prácticas deportivas, y favorece las relaciones interpersonales en espacios públicos exteriores [...] donde estar, sentarse, charlar y relacionarse, todo ello sin necesidad de mediación de ninguna actividad comercial" (Valdivia 2018, 80). Además, promueve la participación ciudadana al desarrollarse múltiples actividades de carácter grupal e individual, las que buscan contribuir en el rediseño físico y simbólico de las ciudades mediante expresiones gráficas, académicas y sociales.

\section{EL CONTEXTO SOCIAL DE LOS ESPACIOS PÚBLICOS DE CIUDAD JUÁREZ}

Ciudad Juárez es una urbe que se distingue por formar parte de la frontera norte de México, lo cual hace que tenga vecindad con los Estados Unidos, específicamente, con la ciudad de El Paso, Texas. La condición de frontera ha ocasionado que Juárez desde la década de 1960 se haya inscrito en un 
proceso de industrialización al desarrollar programas para atraer fuentes de empleos asociados con el maquilado de múltiples piezas. Por ejemplo, en 1961 comenzó a funcionar el Programa Nacional Fronterizo -Pronaf- con la finalidad de elevar el nivel económico, social, cultural y urbano tanto de la frontera norte como la del sur del país mediante la inversión pública y privada. Asimismo, en 1965 se creó el Programa de Industrialización Fronteriza con la intención de 1) desarrollar "mejores niveles de vida para la población fronteriza; 2) mejoramiento del nivel de calificación de la mano de obra [...]; 3) incremento del consumo de insumos nacionales en las operaciones de plantas maquiladoras; y, 4) reducción del déficit comercial" (Gutiérrez 2009, 146).

Los programas generaron que Juárez se adentrara en un proceso de transición económica al alejarse de una economía basada en el sector primario para centrarse en dinámicas de producción terciaria. Ello, produjo un cambio en la concepción de la urbe al asociarla con una zona de desarrollo y de progreso económico debido a que acondicionó los espacios para mejorar las condiciones de producción, distribución y circulación de las actividades fabriles. Es decir, se construyeron parques industriales dentro de la mancha urbana y cerca de los cruces fronterizos. Así como, vialidades y puentes que conectan con las avenidas principales. Sin embargo, se carece de "la infraestructura necesaria [para la convivencia] al igual que normas reguladoras donde se considere a los habitantes y las actividades de éstos dentro de la ciudad" (Gutiérrez 2010, 4).

La industrialización ocasionó una configuración desigual del territorio al crear mayor infraestructura para la industria maquiladora que se asentó al norte y al centro de la localidad, lo cual generó procesos de segregación social porque favoreció a un bajo sector de la población y aumentó las carencias en los servicios públicos dentro de las periferias. Al respecto, Giglia $(2014,161)$ señala que las zonas menos privilegiadas se distinguen por "el descuido y abandono crónico en que se encuentran las calles, las banquetas y los parques en la mayor parte de la ciudad, así como la falta de alumbrado".

La dicotomía urbana ha configurado a Juárez como un espacio excluyente que no permite el desarrollo de diversos usos sobre la infraestructura urbana, ya que está destinada a los procesos de producción industrial que benefician la circulación vial e imposibilitan la creación de dinámicas de interacción social, de movilidad y de conectividad entre los habitantes. Además, las problemáticas sociales como los feminicidios y la violencia sistémica excluyen a la ciuda- 
danía de los espacios públicos porque generan percepciones de inseguridad, lo cual los aleja de los lugares.

La percepción de inseguridad ha afectado a los hombres, pero, sobre todo a las mujeres porque han sido vulneradas en los espacios de Juárez puesto que fueron "abordadas y secuestradas mientras recorrían el espacio público. De la misma forma, sus cuerpos ultrajados suelen encontrarse abandonados en algún lugar poco frecuentado del espacio público" (Ibíd., 161). Entonces, las calles, las plazas y los parques se presentan a las mujeres como entidades urbanas que participan en la vulnerabilidad social de la integridad física y simbólica de la figura femenina, ya que han sido el objeto de ataques desde el año de 1993 cuando se comenzaron a registrar oficialmente los feminicidios.

En este sentido, Giglia $(2014,162)$ señala que los feminicidios ocasionaron que la mujer fuera relegada al ámbito privado porque la figura femenina se limita a moverse en el "propio barrio y a un radio de circulación menor. [...] Además, no sólo se mueven menos, sino que a menudo lo hacen en compañía de algún familiar y no solas". Asimismo, la autora menciona que los lugares que prefieren frecuentar son los que les brindan cierta seguridad como los centros comerciales. Por lo tanto, es necesario pensar en la configuración de los espacios de manera más inclusiva para que permitan la diversidad identitaria y las demandas colectivas de la ciudadanía.

El carácter inclusivo para los espacios públicos de Juárez es una noción que implica la participación de los diferentes actores que conforman a la sociedad, ya que supone un esfuerzo desde el ámbito político, comercial y cultural para la integración de las posturas teóricas y empíricas que posibilitan la transformación simbólica, pero, sobre todo física del espacio fronterizo. Por ejemplo, las perspectivas feministas y de género invitan a pensar en la construcción de los espacios a partir de colocar a la población como elemento primordial para que grupos minoritarios utilicen las calles, las plazas o los parques dependiendo de las necesidades identitarias de los ciudadanos. Esto conlleva a la configuración de un espacio en el que se incluyan las actividades productivas y reproductivas para la facilitación de dinámicas de actuación a nivel individual, familiar o industrial. 


\section{LAS PARTICULARIDADES DE LA ESCENA FEMENINA DEL GRAFITI, STREET ART Y MURALISMO URBANO DE CIUDAD JUÁREZ}

La configuración de espacios públicos inclusivos implica la consideración de la sociedad civil para la integración y contemplación de las diversas identidades en los procesos de urbanización de la ciudad. Asimismo, supone la participación de la ciudadanía en el desarrollo de esfuerzos individuales o colectivos que propicien dinámicas de transformación del espacio. De ello, surge una pregunta ¿cuáles son las identidades urbanas de Ciudad Juárez que colaboran en el cambio de actividades que se producen dentro de los espacios públicos para resignificarlos social, física o simbólicamente? Una respuesta se encuentra en la práctica de la gráfica urbana femenina de la urbe fronteriza, ya que hacen uso del grafiti, street art y muralismo para manifestarse acerca de la urbanización y de las problemáticas sociales que se generan en la estructura urbana de Juárez.

En este sentido, la escena femenina del grafiti, street art y muralismo urbano de Ciudad Juárez se distingue por ser una práctica naciente al conformarse por un número reducido de mujeres que intervienen gráficamente los espacios públicos. Por ejemplo, se encuentra estructura por Mala, Lovely, Poli no police, Nema y batallones femeninos quienes tienen seis y siete años activas dentro del movimiento, lo cual las convierte en las jóvenes con mayor experiencia. Asimismo, participan chicas como Flak, Ema, Alma, Majo, Kikiramen, Amairani y Naye que se integraron a finales de 2018 a la actividad de pegar piezas en las calles. También, se observa un grupo de mujeres que son integrantes de la Cholombia, las que emplean la técnica del esténcil para manifestarse públicamente en contra de los feminicidios y de las problemáticas de la urbe.

Asimismo, la escena se caracteriza por la participación de mujeres jóvenes que poseen una edad de entre los 22 a los 27 años, así como, por tener estudios de nivel superior asociados con las ciencias sociales. También, son estudiantes universitarias de los programas de diseño gráfico y artes visuales, $y$, algunas tienen carreras trunca en historia y ciencias de la comunicación. Además, tienen diferentes trabajos dentro de estudios de tatuajes, en dependencias del gobierno y en la impartición de talleres vinculados con la práctica del paste up. 
La participación de la mujer en los movimientos gráficos-callejeros de Juárez posee características propias que la diferencian de otros contextos de actuación. Por ejemplo, las participantes se distinguen, en primer lugar, por la postura feminista que asumen las integrantes, lo cual se refleja tanto en su actitud como en la estructura sintáctica y semántica de las piezas gráficas. Es decir, las ideas sobre el feminismo son trasladadas a las propuestas que realizan en las calles puesto que con las intervenciones "se rebelan contra aspectos particulares de su condición y manifiestan las situaciones que viven y perciben como injustas; [...] la voluntad de actuar colectivamente contra el sistema de prohibiciones y exclusiones que las encierra en identidades impuestas" (Montero 2006, 169).

En segundo lugar, se distingue por un marcado activismo social que integran en los contenidos discursivos de las piezas gráficas para la realización de críticas sociales sobre la violencia de género que impera en Ciudad Juárez. También, buscan producir un cambio social y político respecto a la problemática de los feminicidios. Así como, alertar a la comunidad femenina de los actos delictivos dirigidos a las mujeres.

En tercer lugar, se caracteriza por la necesidad de desarrollar una escena colectiva que permee las prácticas del grafiti, street art y muralismo femenino dentro de Ciudad Juárez para la creación de una dinámica que acepte la diversidad y las capacidades de las identidades femeninas. Con ello se busca erradicar los procesos de competitividad y rivalidad que afectan la participación de las mujeres en las actividades gráficas para lo cual se pretende estimular actitudes que favorezcan el compañerismo, la unión y la solidaridad que desembocan en acciones sociales que nutren técnica e intelectualmente a las participantes.

\section{LA EXPERIENCIA URBANA DE LAS GRAFITERAS, ARTISTAS Y MURALISTAS EN LOS ESPACIOS PÚBLICOS DE CIUDAD JUÁREZ}

La incursión de Poli no Police, Nema, Mala y Lovely en las dinámicas del street art se debió a dos aprendizajes que definieron su actual forma de intervenir y de comprender la ciudad. Por un lado, se produjo un cambio en la postura ideológica que asumían al dejar una actitud indiferente o pasiva para posicio- 
narse al interior de las perspectivas feministas y anarquistas para "subvertir arraigados códigos culturales, normas y valores, así como el sistema simbólico de interpretación y representación que hace aparecer normales comportamientos y actitudes sexistas" (Montero 2006, 171).

Por otro lado, entendieron al espacio público de Ciudad Juárez como una entidad incapaz de brindar seguridad a las personas, ya que en la urbe se desarrollan varias problemáticas sociales que afectan la interacción de los habitantes. Además, consideran que no cuentan con la infraestructura necesaria para que se fomente su utilización entre los habitantes al carecer de un diseño urbano adecuado, de mantenimiento, de alumbrado público y de garantías sociales. Por lo que señalan que los espacios no tienen la capacidad de transfigurarse en lugares de encuentro que posibiliten dinámicas de socialización, así como, reuniones sociales debido a la inseguridad que hay en la ciudad.

El espacio público es muy deficiente, la verdad, no es como que no haya espacios públicos que se puedan desarrollar, más bien, no los desarrollan bien, no hay seguridad. O sea, no es que no los haya, simplemente en la forma en que los hay es una forma en que la gente no se pueda sentir segura para usarlos como los parques o estos lugares de reunión, como hasta los no lugares.

(Nema en conversación con el autor, 12 agosto 2019)

La concepción de inseguridad sobre los espacios públicos ocasionó que Poli, Nema, Mala y Lovely tomaran mayor conciencia acerca de las problemáticas sociales, pero, principalmente de la misoginia que impera en la sociedad fronteriza, ya que han vivido actos de acoso de manera habitual por las calles de la urbe. Esto, ha generado que el transitar cotidiano de ellas por los espacios públicos sea interpretado como un acto de hostigamiento al estar lleno de vituperios, piropos, miradas lascivas, silbidos, gestos vulgares, así como, ser víctimas de persecuciones. El acoso, produjo que desarrollaran un estado de alerta mental sobre su entorno y de sí mismas para responder a las situaciones de peligro que les supone los múltiples espacios de Ciudad Juárez. Al respecto, Mala señala que la experiencia urbana que tiene de la ciudad no es placentera, más bien, se resume en una dinámica caótica de circulación por las calles.

Andar bien trucha siempre, bien alerta y en guardia porque en lo personal yo sufro de acoso todos los días, cada vez que salgo a la calle es estar cuidándome de los carros, de los batos que me gritan cosas, de los batos que se me acercan, que me siguen, hombres 
todos, eh, y lo aclaro, porque creo necesario aclarar que el acoso viene por esa parte. A cualquier hora del día, ni siquiera en plena luz del día una se siente segura de andar caminando en la calle, sin pensar que un carro la vaya a levantar, en realidad el andar cotidiano para mí no es placentero ni lo disfruto, es un estado de alerta constante.

(Mala en conversación con el autor, 12 agosto 2019)

La percepción de inseguridad ocasionó que desarrollaran estrategias tanto para la estancia dentro de los espacios públicos como para la defensa personal ante cualquier situación de peligro en la urbe. Por ejemplo, cuando Nema recorre a píe las calles opta por caminar por los camellón, circular en sentido contrario, simular que conoce la zona por la que transita y salir acompañadas de otras personas. Además, han decidido esconder en sus pertenencias gas pimienta, táser u objetos cotidianos para salvaguardar su integridad personal. La decisión busca preparar psicológicamente a las chicas para estar protegidas ante las adversidades sociales. Es decir, la posesión de objeto de defensa personal se convierte en un acto proteccionista al brindar cierta seguridad a las mujeres, ya que puede impedir o repeler agresiones.

Yo tengo navajas, tengo picos, tengo gas pimienta, tengo táser, tengo como por cada cosa dependiendo de cuál situación voy hacer. Entonces, cargo el arma que se me facilita más para usar en medio de emergencias. Es como un constante estar pensando para dónde vas a correr, es mucha presión, te crea ansiedad, muy incómodo.

(Nema en conversación con el autor, 12 agosto 2019)

La violencia de género que se ha desarrollado en Ciudad Juárez afecta a las mujeres de diferentes maneras, por ejemplo, se observan dinámicas domesticas de carácter violento, así como, actos asociados con el acoso callejero, laboral, sexual, violaciones, persecuciones, secuestros y asesinatos. Estas acciones, según Paola Gamboa (2019) han aumentado en las últimas décadas al sumar más de 1700 feminicidios desde 1993, lo cual se refleja en las denuncias de agresiones dirigidas a la mujer. Parafraseando a Gamboa (2019) entre los años 2014 al 2016 se registraron alrededor de 200 denuncias, pero, para el año 2018 se contabilizaron 816 agresiones. Asimismo, en los primeros cinco meses del 2019 se han "denunciado ante la Fiscalía Especializada en Delitos de la Mujer 94 casos de violación y 64 de abuso sexual. [...] Durante este 2019 se han cometido 55 asesinatos contra mujeres, además que tanto violaciones, violencia física y feminicidios" (Gamboa Paola 2019, s/p). 
Los datos y las experiencias sobre la violencia contra la mujer han generado en Poli no Police, Nema, Mala y Lovely que posean una percepción negativa acerca de los espacios públicos de Ciudad Juárez al considerar que alejan a las mujeres de su utilización. Esto, es entendido como una dinámica de exclusión social y de desatención a los procesos de socialización de los grupos minoritarios al interior de la sociedad, particularmente el femenino. El desinterés político limita el acceso a los espacios abiertos debido a las constantes acciones violentas dirigidas a la mujer. Así como, a la omisión del Estado para atender de manera eficaz las necesidades y el estado de vulnerabilidad en la que se encuentra el colectivo femenino dentro de la frontera.

La falta de seguridad social para las mujeres ha ocasionado que Poli, Nema, Mala y Lovely consideren que no existen lugares públicos que permitan la presencia segura y libre de la figura femenina en la frontera, ya que no hay tanto un compromiso político como una cultura de respeto en la sociedad. Sin embargo, en el año 2018 se crearon dos espacios semi públicos, el Instituto Municipal de la Mujer y la Casa Centro por 16, las cuales se ubican en la zona centro de Ciudad Juárez. Ambos lugares funcionan con el apoyo del gobierno y están destinados principalmente para la figura femenina. Los espacios se caracterizan por hacer uso de la perspectiva de género para el establecimiento de las relaciones sociales. Así como, por el desarrollo de actividades que fomentan el arte, la estima femenina y el empoderamiento de la mujer. Además, se distingue por la impartición de cursos artísticos, la capacitación social y la dirigencia se encuentra a cargo de mujeres.

Bueno centro por 16 se refiere más a la comunidad femenina y la comunidad LGBT. Una de las características es que son espacios exclusivos para mujeres. Otra, es que se fomentan talleres de defensa personal, de amor propio, donde las mujeres puedan desarrollarse libremente como fiestas sólo para morras. Entonces, es más bien, como en general crear este ambiente donde una se sienta cómoda de ser como es, poder hacer lo que tú quieras porque sabes que nada te va a pasar dentro de ahí.

(Nema en conversación con el autor, 12 agosto 2019)

Poli, Nema, Mala y Lovely señalan que los espacios públicos debería de poseer las diferentes características institucionales que tienen tanto la Casa Centro por 16 como el Instituto Municipal de la Mujer para que empezaran a prevalecer dinámicas de respeto en las calles. Además, consideran que es necesario un cambio social y cultural en la sociedad para atender de raíz la problemática de la violencia de género dentro de Ciudad Juárez, lo cual ayu- 
daría a transformar actitudes machistas en posturas basadas en la tolerancia y en la diversidad. La idea del cambio de paradigma busca la cimentación de nuevos valores al interior de la sociedad fronteriza que permitan avanzar en las dinámicas de socialización entre los hombres y las mujeres. Así como, erradicar las relaciones de subordinación que afectan a la figura femenina en distintos ámbitos de la vida.

Yo siento que los espacios deberían tener una planificación con una vista a lo que es la perspectiva de género para su creación y su implementación, porque si no existe está educación de perspectiva de género, no va a cambiar aunque le pongas rosa a las paredes, Entonces, yo siento que los espacios públicos tienen que ser gestionados e implementados por personas que estén capacitadas y comprometidas a generar espacios seguros.

(Lovely en conversación con el autor, 12 agosto 2019)

\section{LA FEMINIZACIÓN GRÁFICA DE LOS ESPACIOS PÚBLICOS DE CIUDAD JUÁREZ}

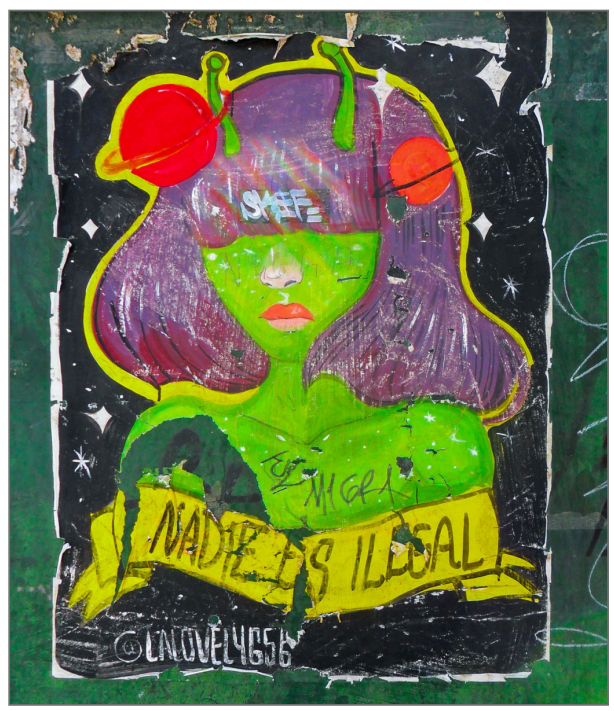

Fig. 1. Pieza de paste up elaborado por Lovely. La propuesta hace alusión a la migración. Av. Juárez. Ciudad Juárez. Año 2019. Fotografía: Raúl Recio.
Poli no Police, Nema, Mala y Lovely junto con Amairani, Majo, Nayely, Flak y Ema han participado en los movimientos callejeros bajo la perspectiva feminista que les ha permitido que entiendan de manera crítica su estancia dentro de los espacios públicos y las dinámicas sociales de Ciudad Juárez. La actitud crítica ha sido trasladada a las piezas del paste up debido a que han estructurado las composiciones con temáticas alusivas a la posición que posee la mujer en la frontera. Por ejemplo, han elaborado carteles, lettering, grafitis y murales referentes a las problemáticas que las aquejan cotidianamente como el acoso y la violencia. Ello, ha sido empleado para participar en la feminización de los espacios públicos fronterizos. 
La participación en la feminización de los espacios ha sido posible a la creación de piezas gráficas que se cimentan en la realidad social de Ciudad Juárez puesto que son estructuradas por temáticas que hacen alusión a las identidades femeninas que existen dentro de la sociedad al ser el elemento discursivo y conceptual que posibilita la mostración de la diversidad de cuerpos femeninos al pintar rasgos físicos alejados de los estereotipos de belleza eurocentrista que excluyen a las jóvenes que no cumplen con los cánones estéticos.

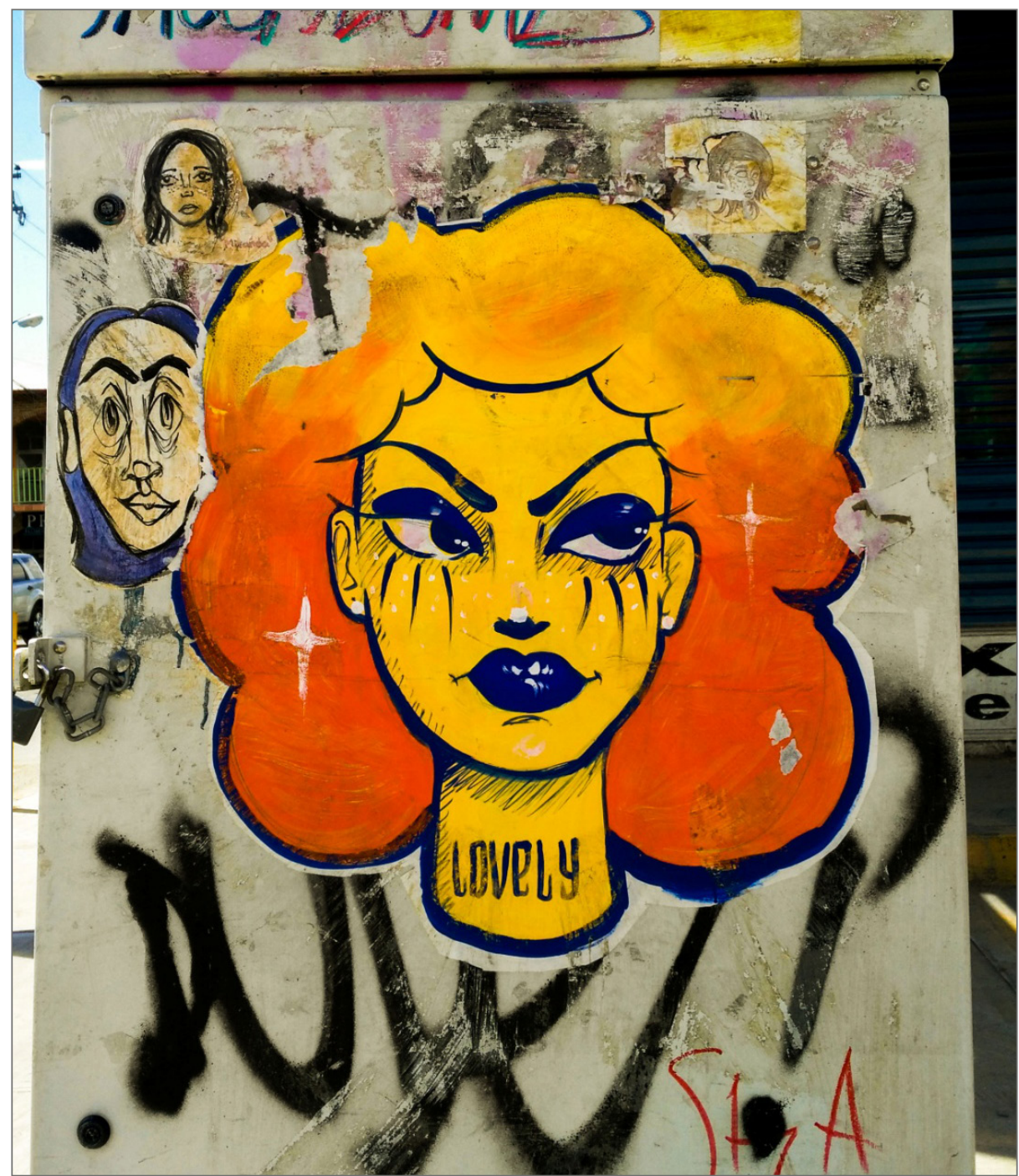

Fig. 2. Ejemplo de identidades femeninas. Autor: Lovely. Av. Valentín Fuentes. Ciudad Juárez. Año 2019. Fotografía: Raúl Recio. 
Además, representan las actividades que elaboran las mujeres en sus vidas cotidianas para abordar las personalidades de indígenas, trabajadoras, activistas, estudiantes, peatonas y migrantes. Aquí, la imagen de la mujer sirve para la realización de una crítica social que evidencia una desaprobación sobre la permanencia de actitudes retrógradas que incitan a la segregación social dentro de Ciudad Juárez.

Asimismo, en las piezas gráficas se integra la figura femenina como elemento trascendental de las composiciones al ser el sujeto que importa representar para cambiar diferentes ideas y estereotipos que promueven la inequidad de género que alejan a la sociedad de formas de vida justas y ecuánimes. De ahí que las artistas busquen otorgarles una subjetividad, voz y poder a las mujeres para que sirvan de símbolo de identificación entre el colectivo femenino. Aquí, se transfigura a la mujer en un cuerpo discursivo que hace alusión a la opresión, esclavitud y violencia, así como, al pensamiento, a la esperanza, y a las virtudes. Entonces, la representación de la mujer "se trata sólo de un proceso subversivo, una dinámica a la vez productiva y amenazadora, productiva porque las mujeres empiezan a tomar el mando de sus fantasías, amenazadora (para el Establishment) porque la mujer aparentemente inmutable [...] ha echado andar" (Lenk 1986, 60).

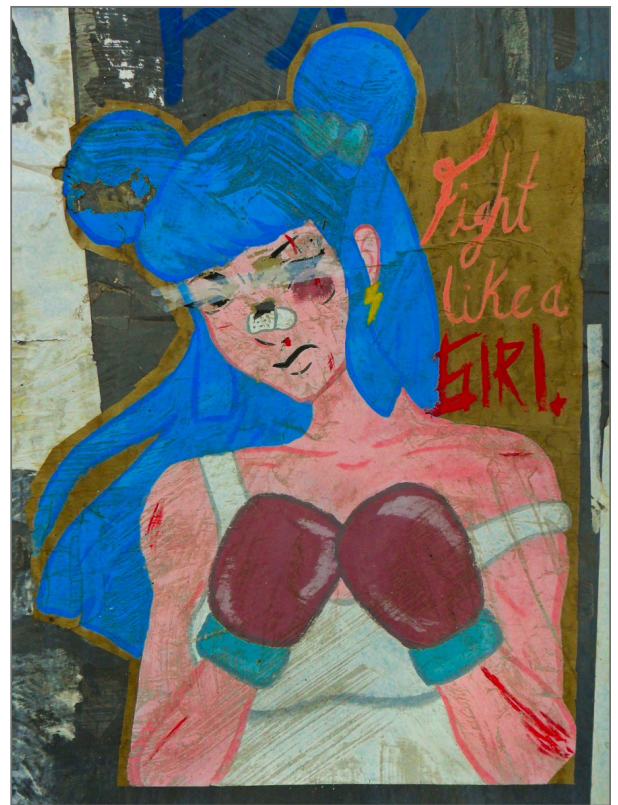

La figura de la mujer es muy importante, ya que tratamos de hacer distintos tipos de mujeres para ese mismo estereotipo de la figura femenina, alimentarlo y que salga más allá de lo que ya está hecho ¿no? Este, a mi me gusta poner una chica que parezca inofensiva o que a primera vista la ves y es algo muy bonito y todo esto, te llama la atención porque es amigable a la vista, lo ves y te está regañando, te está diciendo que no acoses o algo así. Entonces, si es muy importante darle poder a la figura femenina.

(Lovely en conversación con el autor, 12 agosto 2019)

Fig. 3. Pieza de paste up. Av. Juárez. Ciudad Juárez. Año 2019. Fotografía: Raúl Recio. 
Entonces, participan en la feminización de los espacios públicos mediante la creación de mensajes dirigidos a las mujeres, ya que las alerta sobre la misoginia social que reproduce actitudes machistas al interior de la sociedad de Ciudad Juárez. Aquí, las propuestas se erigen como escenas reflexivas que muestran el descontento con los procesos patriarcales al visibilizar consignas que enfrentan a los agresores. Además, exponen las capacidades intelectuales del colectivo femenino en los diferentes

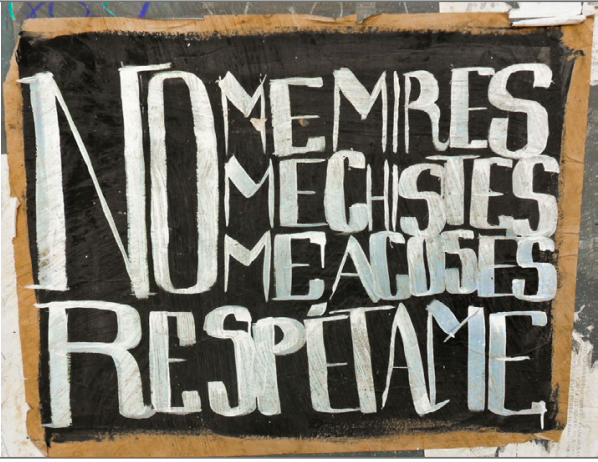

Fig. 4. Pieza de paste up. Avenida Juárez. Ciudad Juárez. Año 2019. Fotografía: Raúl Recio. ámbitos del mundo social.

Las integrantes han entendido su participación en la feminización como un esfuerzo minúsculo puesto que se enfrentan, por un lado, a la problemática de la violencia de género que las ha condenado a un ostracismo dentro del territorio de Ciudad Juárez debido a la perpetuación de acciones que quebrantan la personalidad femenina. Por otro lado, se afrontan al arraigo cultural del sistema patriarcal que domina las múltiples esferas del ámbito social como la familia, la iglesia y el Estado. Sin embargo, consideran que sus intervenciones gráficas son actos significativos para la sociedad al buscar la concientización de las personas sobre la importancia de la mujer dentro de la vida cotidiana y de los comportamientos negativos de los hombres que desmeritan la presencia de la figura femenina en la urbe.

Por eso te digo, el arte callejero es una respuesta a eso, es empoderarte, salir a las calles e intervenirla, apropiarte de las calles porque es algo que a veces hasta te da miedo. Entonces, si es como un empoderamiento y voy a salir y voy a quitarme ese miedo y voy hacer algo en las calles.

(Poli no pólice en conversación con el autor, 12 agosto 2019)

La participación de las mujeres en la feminización de los espacios es una forma de actuación subversiva que busca cambiar las lógicas machistas mediante la comunicación de mensajes tipográficos y figurativos sobre las capacidades que poseen las mujeres en los distintos ámbitos de la sociedad. Además, es un acto que pretende la reconfiguración de los aprendizajes sociales al interior de los sistemas patriarcales porque ofrece ideas alternativas de actuación asociadas con la estima personal. Ello, lo consiguen a través de la apropiación 
del paisaje urbano y social de la ciudad para "contribuir a su transformación, o al menos, en las personas que la transitan, proponiendo imágenes diferentes que favorezca, en este caso, la igualdad de género, o de sexos, a través de la cultura visual colectiva" (Luque 2018, 80).

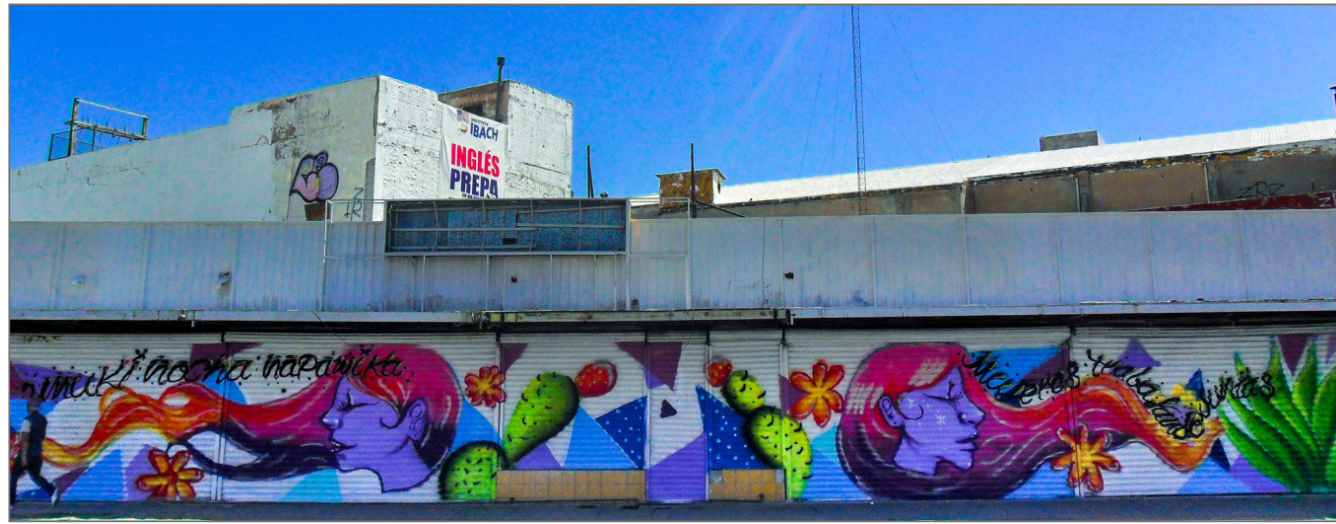

Fig. 5. Mural elaborado por el colectivo Morras pintando muros. La propuesta hace alusión a la unión de las mujeres. Av. Francisco Villa. Ciudad Juárez. Año 2019. Fotografía: Raúl Recio.

La feminización de los espacios es una acción combativa que enfrenta de manera gráfica al colectivo social que no permite la superación de los procesos androcéntricos que descalifican y colocan a las mujeres en situaciones de riesgo dentro de Ciudad Juárez. Las propuestas se presentan en los espacios públicos para contradecir los discursos oficiales y las representaciones sociales que buscan ocultar la violencia de género y los problemas que giran en torno a la figura femenina de la frontera. De ahí que la actividad gráfica trate de "hacer visibles las vías que las mujeres han empleado para (re)apropiarse de los espacios y a través de ellos subvertir el orden simbólico masculino" (Pérez 2013, 95).

Las piezas de paste up se convierten en signos de alerta porque avisan al colectivo femenino de los peligros que suponen la estancia temporal de la mujer en los espacios abiertos, así como, de los riesgos que pueden sufrir al interior del ámbito privado. Ello, busca la construcción de una consciencia colectiva que desemboque en el aumento de relaciones sociales de sororidad entre las mujeres para la disminución de acciones violentas provenientes del patriarcado. Por lo tanto, los mensajes se erigen como formas discursivas que cuestionan la normalización de las actitudes machistas que condenan a la mujer a vivir procesos de inseguridad. 
A mí me gusta pintar tags, el tag es una firma, bombas, me gusta pintar morras, nos gustan frases, letras, cosas que tengan que ver con resistencia, me gusta pintar sobre el acoso que nos caga, tratamos de alertar a las morras con mensajes, así como de ¡Ponte vergas! ¡Trucha! ¡Cuídate!

(Mala en conversación con el autor, 12 agosto 2019)

Las composiciones del paste up aparecen en la ciudad como entidades discursivas que pretenden generar relaciones dialógicas con el espectador para producir dinámicas comunicacionales y de actuación en la sociedad que hagan frente al panorama negativo que experimentan las mujeres dentro de Juárez. Además, en las piezas gráficas se despliegan las identidades de las grafiteras, las artistas y las muralistas, ya que son el espacio en el que desarrollan una "nueva relación consigo misma a través de sus relaciones con otras mujeres. La mujer se convertirá en el espejo viviente de la mujer en el que se pierde así misma para encontrarse" (Lenk 1986, 67). Por lo tanto, las obras funcionan como signos de identificación que fortalecen simbólicamente al colectivo femenino para desarticular los procesos de marginación histórica que han construido actitudes peyorativas respecto a la figura femenina.

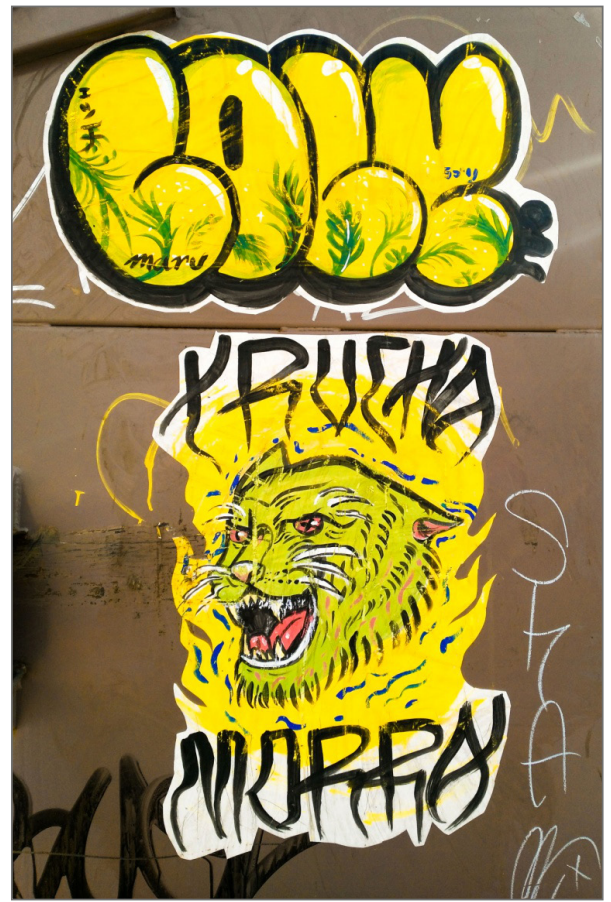

Fig. 6. Paste up de Mala. Mensaje de alerta dirigido hacia las mujeres. Simona Barba. Ciudad Juárez. Año 2019. Fotografía: Raúl Recio.

Los espacios feminizados simbólicamente son los lugares que transitan con mayor frecuencia las integrantes debido a que han colocado piezas de paste up en las áreas cercanas al hogar, al trabajo, al entretenimiento y al aprendizaje. Además, emplean zonas con mayores estigmas sociales sobre la violencia hacia la mujer, es decir, han intervenido con mayor frecuencia el centro histórico de Ciudad Juárez puesto que es una área en la que las mujeres han quedado expuestas ante actos delictivos. Aquí, las propuestas cobran mayor relevancia e identificación porque avisan a la mujer sobre los riesgos que poseen los lugares. 


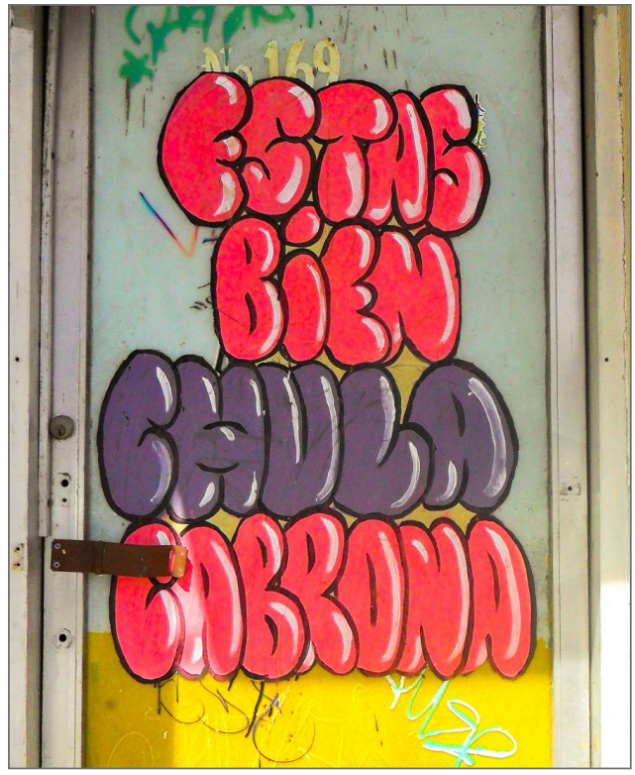

Fig. 7. Paste up elaborado por Nema. Mensaje que cuestiona los cánones de belleza e invita a la mujer que se sienta bella. Ciudad Juárez. Año 2019. Fotografía: Raúl Recio.

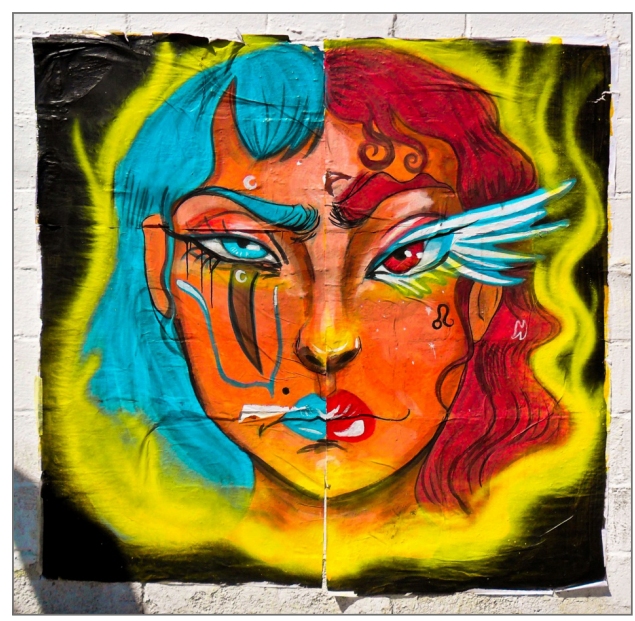

Fig. 8. Pieza de paste up elaborada por Lovely. La propuesta hace alusión a la desaparición de Dana. Av. Del Charro. Ciudad Juárez. Año 2019. Fotografía: Raúl Recio.
La participación de las artistas en la feminización de los espacios se limita a un nivel simbólico debido a que cuentan con escasos recursos culturales, económicos y políticos que no les permiten realizar cambios macro-estructurales en el entorno urbano de Juárez. Por lo tanto, no se presentan transformaciones sustanciales en los espacios; más bien, la feminización pasa por un proceso comunicativo donde manifiestan su determinación y su fortaleza para abordar la conversión de la imagen de la mujer en la sociedad y disminuir la misoginia que coarta la libertad ciudadana.

Los mensajes aportan a la visibilización de los problemas en la comunidad de mujeres porque la feminización del espacio no siento que sea algo que podría decir. Utilizamos elementos gráficos considerados femeninos para atrapar la atención porque es algo que la gente ve. Yo siento que si ayuda porque a su vez ayuda a que más mujeres no se sientan avergonzadas de que les guste algo que les han puesto en sus mentes desde hace un chingo ¿no? Pero a su vez también te cuestiona.

(Lovely en conversación con el autor, 12 agosto 2019)

Asimismo, la participación de las artistas no se produce desde el ámbito gubernamental, lo cual genera que la contribución a la feminización de los espacios se desarrolle desde un 
entorno personal y colectivo que aboga por la autonomía social de las mujeres al alejarlas de las prescripciones que limitan su comportamiento. De ahí que los contenidos discursivos nieguen los valores sociales impuestos por la cultura dominante al impugnar todas las expresiones surgidas del sistema patriarcal. De hecho, la feminización gráfica implica otra forma de ver la realidad, ya que propone una nueva mirada sobre la condición de la mujer donde le permite que prepondere la subjetividad que se construye de sus necesidades y cualidades.

Entonces, las acciones con las que se materializa la feminización gráfica de los espacios de Juárez se asocian con la colocación de piezas de paste up y la elaboración de murales, los cuales contienen mensajes críticos sobre la opacidad política y social que posibilita el desarrollo de acciones violentas contra las mujeres. Las propuestas cumplen con la función informativa de exponer de forma breve la perspectiva de género y los postulados del feminismo, ya que señalan demandas acerca de la necesidad de elección que debe tener la figura femenina en la urbe fronteriza. Además, muestran ideas que enaltecen la condición de la figura femenina al realizar expresiones en torno a las cualidades físicas, intelectuales, sentimentales y biológicas que poseen.

\section{CONCLUSIONES}

La feminización gráfica de los espacios públicos de Ciudad Juárez es una acción social que sugiere una transformación de la urbe que deja atrás una perspectiva de urbanización para atribuirle nuevos valores al territorio fronterizo. Sin embargo, la realidad del proceso de feminización en el que participan Poli no Police, Nema, Mala y Lovely sugiere que no se presenta un cambio sustancial en los espacios debido a la falta de recursos sociales y económicos para la realización de actividades y el involucramiento de personas que apoye la iniciativa de las artistas.

Sin embargo, es un esfuerzo social que se produce fuera de los circuitos institucionales y gubernamentales al surgir desde la práctica del grafiti, del street art y del muralismo. Ello, ha generado que las propuestas gráficas hayan sido estructuradas a partir de la incorporación de mensajes, elementos figurativos y tipográficos que se alejan de las posturas oficiales del Estado para centrarse en ideas que brotan de la experiencia de las artistas y de la ciudadanía. Por 
lo tanto, cuestionan tanto la normalización de la sociedad como la indiferencia del gobierno hacia la violencia de género que se desarrolla en Ciudad Juárez.

\section{Referencias bibliográficas}

De Simone, Liliana Rosa. 2018. "Mujeres y ciudades. Urbanismo género-consciente, espacio público y aportes para la ciudad inclusiva desde un enfoque de derechos". En El estado y las mujeres: El complejo camino hacia una necesaria transformación de las instituciones, Javiera Arce Riffo, 229-50. Providencia, Santiago de Chile: RIL

Gamboa, Paola. 2019. "Se disparan violaciones y abuso sexual contra mujeres y niños". El Mexicano, 29 mayo. https://www.elheraldodejuarez.com.mx/policiaca/se-disparan-violaciones-yabuso-sexual-contra-mujeres-y-ninos-3688177.html

Giglia Ciotta, Ángela. 2014. "Espacio público y género en Ciudad Juárez, Chihuahua". Alteridades 24(48): 161-4. http://www.scielo.org.mx/scielo.php?script=sci_arttext\&pi$\mathrm{d}=\mathrm{S} 018870172014000200015$

Gutiérrez Casas, Luis Enrique. 2009. “Ciudad Juárez en los sesenta: La estructura urbana en transición”. Nóesis 18(36): 128-54. https://www.redalyc.org/articulo.oa?id=85919840007

Gutiérrez Luna, Susana. 2010. “Espacios públicos en la frontera: Estudio comparativo Ciudad Juárez Chihuahua - El Paso Texas". En Ponencias en extenso: VI Congreso Internacional Ciudad y Territorio Virtual, Mexicali, Baja California, 5, 6 y 7 de octubre, 1-15. Mexicali: Universidad Autónoma de Baja California

Lamas Encabo, Marta. "Diferencias de sexo, género y diferencia sexual”. Cuicuilco 7(18): 1-24. https://www.te.gob.mx/genero/media/pdf/45a9a76d6fde4c4.pdf

Lenk, Elisabeth. 1986. “La mujer, reflejo de sí misma”. En Estética feminista, Gisela Ecker, ed.; trad., Paloma Villegas, 59-68. Madrid: Icària

Luque Rodrigo, Laura. 2018. "Graffiti e igualdad de género: La artista Icat y el proyecto educativo del IES Trasiera (Córdoba)". AusArt: 73-82. https://doi.org/DOI: 10.1387/ausart.19156

Milena Montoya, Ana. 2012. "Mujeres, derechos y ciudad: apuntes para la construcción de un estado del arte desde el pensamiento y la teoría feminista". Territorios 27: 105-23. https:// revistas.urosario.edu.co/index.php/territorios/article/viewFile/2282/1987

Montero Corominas, Justa. 2006. "Feminismo: Un movimiento crítico". Intervención Psicosocial 15(2): 167-80. http://scielo.isciii.es/scielo.php?script=sci_arttext\&pi$\mathrm{d}=\mathrm{S} 1132-05592006000200004$

Nadurille Álvarez, Angélica. 2017. "Urbanismo feminista o la respuesta a la emergencia urbana". "Ciudades seguras para mujeres y niñas", número monográfico Dfensor 1(15): 37-9. https://cdhcm.org.mx/wp-content/uploads/2014/05/dfensor_01_2017.pdf

Ortiz Escalante, Sara. 2017. “Urbanismo desde la perspectiva de género: Buenas prácticas con perspectiva de derechos humanos". "Ciudades seguras para mujeres y niñas", número monográfico Dfensor 1(15): 5-9. http://www.corteidh.or.cr/tablas/r36100.pdf

Otero Carrasco, Inmaculada. 2012. "Mercados de género y procesos de feminización en la cultura del consumo: Aproximaciones". En Investigación y género, inseparables en el presente y en el futuro: IV Congreso Universitario Nacional "Investigación y género", Sevilla, 
21 y 22 de junio de 2012, coord., Isabel Vázquez Bermúdez, 1399-1410. Sevilla: Universidad de Sevilla

Pérez Sanz, Paula. 2013. "Reformulando la noción de 'Derecho a la ciudad' desde una perspectiva feminista". Encrucijadas 5: 92-105

Soto Villagrán, Paula. 2018. "Hacia la construcción de unas geografías de género de la ciudad: Formas plurales de habitar y significar los espacios urbanos en Latinoamérica". Perspectiva Geográfica 23(2): 13-31. http://www.scielo.org.co/pdf/pgeo/v23n2/0123-3769pgeo-23-02-13.pdf

Valdivia Gutiérrez, Blanca. 2018. "Del urbanismo androcéntrico a la ciudad cuidadora". Hábitat y Sociedad 11: 65-84. https://revistascientificas.us.es/index.php/HyS/article/view/5172

Notas

${ }^{1}$ Se empleará el término 'paste up' para hablar de la actividad de pegar con engrudo o pegamentos industriales carteles e ilustraciones sobre la infraestructura urbana.

(Artículo recibido: 24-03-20; aceptado: 06-05-20) 